\title{
RECONSTRUCTING THE GLOBAL DYNAMICS OF ATTRACTORS VIA THE CONLEY INDEX
}

\author{
CHRISTOPHER McCORD \\ Institute for Dynamics, Department of Mathematics, University of Cincinnati \\ P.O. Box 210025, Cincinnati, Ohio 45221-0025, U.S.A. \\ E-mail: chris.mccord@uc.edu
}

\begin{abstract}
Given an unknown attractor $\mathcal{A}$ in a continuous dynamical system, how can we discover the topology and dynamics of $\mathcal{A}$ ? As a practical matter, how can we do so from only a finite amount of information? One way of doing so is to produce a semi-conjugacy from $\mathcal{A}$ onto a model system $\mathcal{M}$ whose topology and dynamics are known. The complexity of $\mathcal{M}$ then provides a lower bound for the complexity of $\mathcal{A}$. The Conley index can be used to construct a simplicial model and a surjective semi-conjugacy for a large class of attractors. The essential features of this construction are that the model $\mathcal{M}$ can be explicitly described; and that the finite amount of information needed to construct it is computable.
\end{abstract}

1. Introduction. The Conley index theory has grown and matured substantially in recent years, in both its computational and theoretical aspects. The theoretical developments have deepened the dynamical information that can be extracted from the index; while the computational improvements have broadened the range of applications in which the index can be computed. In this note, I will consider a combination of recent theoretical and computational developments - a combination that I believe points the way to a rich new realm of applications of the index ideas.

The computational developments I refer to are the ongoing efforts to computerize the index computations. As several papers in this volume describe, it is becoming feasible to input a dynamical system (either continuous or discrete) to a computer, and obtain as the output

- An isolating neighborhood;

- A Morse decomposition of its maximal invariant set;

1991 Mathematics Subject Classification: Primary 58F12; Secondary 54H20.

Key words and phrases: attractor, Conley index, semi-conjugacy, simplicial complex.

The author was supported in part by grants from the National Science Foundation and the Charles Phelps Taft Memorial Fund.

The paper is in final form and no version of it will be published elsewhere. 
- The homology Conley index of each of the Morse sets;

- The connection matrix of the Morse decomposition.

Indeed, it is possible to carry out such computations even if the system is only defined by experimental data [14] with no formulaic description at all. While there is still considerable work required to make these index computations a practical reality, the potential (and more than just potential) is clearly there.

But, how can this computational power be exploited? If $\phi$ is a flow (or semi-flow) on a space $X$, perhaps defined by a formula, perhaps only known experimentally, how can we use the ability to compute the Conley index information to understand the dynamics of $\phi$ ? Conley's celebrated decomposition theorem [2] suggests one direction. If $S$ is a compact invariant set in a continuous dynamical system, Conley's theorem states that there is a semi-conjugacy from $S$ onto a gradient-like system. This semi-conjugacy is formed by identifying each component of the chain recurrent set to a point. While this is a powerful structure theorem, its practical utility is limited by the fact that the semi-conjugacy and the gradient-like system are existential. The theorem gives no method for describing or understanding their structure, other than to first understand the structure of $S$ itself. Obviously, if the goal of the analysis is to understand the global structure of $S$, this is not very useful.

However, using Conley's theorem as motivation, we seek a method to use the Conley index information to explicitly construct a compact space $M$, and explicitly define a flow on $M$, such that there is a surjective semi-conjugacy $f: S \rightarrow M$. The essential questions that must be addressed are:

- How much information about $S$ is needed to construct the model flow and semiconjugacy?

- How complicated can the model flow be?

- How do we guarantee surjectivity?

The first two questions are closely related: the complexity of the model is, in some sense, a measure of the information available about $S$. With no information about $S$, we can construct a semi-conjugacy onto a single (rest) point. With complete knowledge of $S$, we can construct a model flow which is conjugate to $S$.

It is important that we not only construct the model and semi-conjugacy, but that we also know exactly what the image of $f$ in $M$ is. It is only $\operatorname{im}(f)$ that carries information about $S$. Since $\operatorname{im}(f)$ is a compact invariant subset of $M$, if we can identify $\operatorname{im}(f)$, we can discard the rest of $M$. That is, the ability to identify $\operatorname{im}(f)$ is essentially equivalent to requiring $f$ to be surjective.

The first theorem along this line was proved in [9]. There, the Morse decomposition consisted of a collection $M_{0}, M_{1}, \ldots, M_{P}$ with partial order $0<1<\ldots<P$. The homology Conley index of $M_{i}$ was assumed to be that of an orientable hyperbolic periodic orbit with unstable dimension $2 i$ for $i<P$; and that of a hyperbolic fixed point with unstable dimension $2 P$ for $M_{P}$. The Morse sets below $M_{P}$ were each assumed to admit a Poincaré section, and some technical algebraic hypotheses were also assumed. From this information, a Morse-Smale flow on a $2 P$ disk with $P$ peri- 
odic orbits and one rest point, and a surjective semi-conjugacy to the disk, were constructed.

Similar results, with slightly simpler structures, were proved in $[5,6,10,11]$. In [10], for example, the Morse decomposition consisted of $2 P+1$ Morse sets $M_{0}^{+}, M_{0}^{-}, \ldots, M_{P-1}^{+}$, $M_{P-1}^{-}, M_{P}$, with partial order $(0, \pm)<(1, \pm)<\ldots<(P-1, \pm)<P$. The homology Conley indices were those of a hyperbolic fixed point of index $p$ for $M_{p}^{ \pm}$and of index $P$ for $M_{P}$. The connection matrix was assumed to have $\Delta_{q p} \neq 0$ if and only if $p$ and $q$ are adjacent in the partial order. With this structure, a Morse-Smale flow on a $P$-disk with $2 P+1$ rest points and no periodic orbits, and a surjective semi-conjugacy to the disk, were constructed.

It is natural to expect a general theorem that subsumes these results. Such a theorem might be paraphrased as

Mantra If the flow on an isolated invariant set $S$ "looks like" (i.e. has the same Morse decomposition and Conley index invariants as) a Morse-Smale flow on $M$, then there is a semi-conjugacy $f: S \rightarrow M$ that conjugates the index invariants.

This is a "mantra" rather than a "conjecture" because there is good reason to believe that it is not literally true. But it is certainly a useful guiding principle for developing results. One step in this direction was taken in [8]. This note is essentially a summary of the results of [8], together with some speculations on the next steps towards realizing the mantra. The next section states the semi-conjugacy existence and surjectivity results of [8], while $\S \S 3$ and 4 outline the details of the constructions. The last section considers verifiability and necessity of the hypotheses, as well as possible generalizations.

2. Statement of results. Semi-conjugacies are constructed in [8] for attractors in continuous dynamical systems satisfying the following hypotheses:

Ho $\mathcal{A}$ is an attractor in a continuous semi-flow on a locally compact metric space $X$. On $\mathcal{A}$ itself, there is a complete two-sided flow.

H1 $\mathcal{A}$ has a Morse decomposition $\left\{S_{p}\right\}_{p \in P}$ indexed by the partially ordered set $(P,<)$.

H2 Each Morse set $S_{p}$ has the homology Conley index of a hyperbolic rest point. That is, for each $p \in P$, there exists an $n(p)$ such that

$$
C H_{k}\left(S_{p}\right)=\left\{\begin{array}{cl}
Z & k=n(p) \\
0 & \text { otherwise }
\end{array}\right.
$$

H3 There is a unique connection matrix $\Delta(P)$. This matrix has the property that Morse sets $S_{p}$ and $S_{q}$ are adjacent in the flow-defined ordering if and only if the connection matrix entry $\Delta_{q p}$ is an isomorphism.

From the partial order $(P,<)$, we can construct in a natural way a simplicial complex $\mathcal{M}(P,<)$ by creating a simplex for every totally ordered chain in $P$. This simplicial complex admits a flow $\psi: \mathcal{M} \times R \rightarrow \mathcal{M}$ which leaves each simplex invariant and has the vertex set $\left\{M_{p}\right\}_{p \in P}$ as a Morse decomposition. This will be the model flow that is the target of the semi-conjugacy from $\mathcal{A}$. Its crucial feature is that it is constructed directly from the partial order $(P,<)$ - no further information about the topology or dynamics of $\mathcal{A}$ is required. 
Theorem 1. Suppose $\mathcal{A}$ is an attractor with flow $\phi$ satisfying $\mathbf{H 0}-\mathbf{H 3}$. Let $\mathcal{M}(P,<)$ be the simplicial complex generated by the poset $(P,<)$. Then, up to a time reparameterization of $\phi$, there is a continuous semi-conjugacy $f: \mathcal{A} \rightarrow \mathcal{M}(P,<)$. That is, there is a function $\theta: \mathcal{A} \times R \rightarrow R$ which is monotone increasing in $t$ for every $x \in \mathcal{A}$, such that $f \circ \phi(x, \theta(x, t))=\psi(f(x), t)$. This semi-conjugacy preserves the Morse decomposition structure: $f^{-1}\left(M_{p}\right)=S_{p}$ and for every interval $I \subset P, f^{-1}(M(I)) \subset S(I)$.

The time reparameterization is a technicality, and is only introduced to guarantee that if $f(x)=f(y)$, then $f(x \cdot t)=f(y \cdot t)$. The time reparameterization does not change any of the essential dynamical features of the flow on $\mathcal{A}$, so it is not too imprecise to interpret this theorem as "there is a semi-conjugacy from $\mathcal{A}$ to $\mathcal{M}$."

This theorem does not guarantee that the semi-conjugacy is surjective. At this point, it is not clear whether this is a technical shortcoming of the proof, or whether there are examples in which $\mathbf{H 0 - H 3}$ do not produce surjectivity. It is also natural to ask if the model reproduces the Conley index information used to construct it. That is, since $\mathcal{M}(P,<)$ has a Morse decomposition with the same flow-defined ordering, does it also have the same Conley indices for the Morse sets? Does it have the same connection matrix? Is $f_{*}$ a conjugacy between the algebra on $\mathcal{A}$ and the algebra on $\mathcal{M}$ ?

It turns out that the two questions are closely related. Our proof of surjectivity will use the homology Conley index, and it might be conjectured that, if $M_{p}$ and $S_{p}$ have the same homology Conley index for all $p$, then $f$ is surjective. While we cannot prove such a relationship at this point, we can formulate a condition which is very close in spirit to " $M_{p}$ and $S_{p}$ have the same homology Conley index" and which implies both the equivalence of the indices and the surjectivity of $f$.

For every $p \in P$, let $A_{p}=\{q \in P \mid q<p\}$, and $A_{p}^{+}=A_{p} \cup\{p\}$. Let $M\left(A_{p}\right)$ be the subcomplex of $\mathcal{M}(P,<)$ spanned by vertices in $A_{p}$. To guarantee the surjectivity of $f$, we make the assumption

H4 For every $p \in P$, the complex $M\left(A_{p}\right)$ is homeomorphic to the $(n(p)-1)$-sphere.

If $M\left(A_{p}\right)$ is a sphere, then surjectivity is homologically detectable. We use this observation to obtain the following result:

THEOREM 2. If $\mathcal{A}$ is a compact attractor with flow $\phi$ that satisfies $\mathbf{H 0}-\mathbf{H 4}$, then

1. The semi-conjugacy $f: \mathcal{A} \rightarrow \mathcal{M}(P,<)$ is surjective.

2. For every interval $I \subset P, f_{*}(I): C H_{*}(\mathcal{A}(I)) \rightarrow C H_{*}(M(I))$ is an isomorphism.

3. The Morse decomposition $\left\{M_{p}\right\}_{p \in P}$ has a unique connection matrix $\Delta_{M}(P)$, which is conjugate to $\Delta(P)$ via the isomorphism $F=\oplus_{p \in P} f_{p *}: \oplus_{p \in P} C H_{*}\left(S_{p}\right) \rightarrow$ $\oplus_{p \in P} C H_{*}\left(M_{p}\right)$. That is, $\Delta_{M}(P) \circ F=F \circ \Delta(P)$.

There are several important features to these results. First, the hypotheses fit very well with the output of the computerized index calculations. Hypotheses $\mathbf{H 3}$ and $\mathbf{H} \mathbf{4}$, to be sure, are additional burdens, but both are tractable. The multi-valued map methods used to calculate the index invariants can also be employed to verify $\mathbf{H 3}$, while $\mathbf{H 4}$ can be verified from $(P,<)$ either directly by constructing $M\left(A_{p}\right)$, or indirectly (cf. Theorem 3 below). 
The second feature of these results is that, once the partial order $(P,<)$ is known, the complex $\mathcal{M}(P,<)$ can be easily constructed (and property $\mathbf{H} 4$ checked) without further knowledge of $\mathcal{A}$ or its flow required. This ability to construct and explore $\mathcal{M}$ is of central importance. Once constructed, $\mathcal{M}$ serves as a model for the flow on $\mathcal{A}$. If the semiconjugacy is surjective, then the complexity of $\mathcal{M}$ (both in its topology and dynamics) serves as a lower bound for the complexity of $\mathcal{A}$. If $\mathcal{A}$ is known only existentially, or is part of a system defined only by experimental data, then this model may be the only evidence of $\mathcal{A}$ 's structure available. It can then serve as a guide to further investigations of the set.

In sum, a finite amount of information about $\mathcal{A}$ allows a Morse-Smale model flow $\mathcal{M}$ to be explicitly constructed, and to guarantee that the dynamical structures revealed by that model will be a lower estimate for the dynamics on $\mathcal{A}$.

3. The simplicial model. The construction of the simplicial model $\mathcal{M}(P,<)$ is very natural, and the properties of the model are easy to establish. The hypotheses on the Morse decomposition imply that all of the information needed to construct the complex is carried by the poset $(P,<)$. Indeed, the simplicial model $\mathcal{M}(P,<)$ can be thought of as a geometric realization of the partial order $(P,<)$. An inductive construction of this geometric realization is:

1. The elements of $P$ are the 0 -skeleton.

2. Form the 1-skeleton by adding an edge from $p$ to $q$ if $q<p$.

3 . Inductively add the $k$-skeleton by filling in all possible $k$-simplices. That is, if all of the $k-1$ simplices required to form $\partial \sigma$ are present in the $(k-1)$-skeleton, then add $\sigma$ to the $k$-skeleton.

A non-inductive formulation of this [4] is:

Definition 3.1. $\left[p_{0} p_{1} \ldots p_{n}\right]$ is a simplex in $\mathcal{M}(P,<)$ if and only if each $p_{i}<p_{i+1}$. If $I \subset P$ is an interval in $P$, let $M(I)$ denote the maximal subcomplex spanned by the vertices in $I$

That is, $\sigma$ is a simplex in $\mathcal{M}(P,<)$ if and only if there is an ordering of its vertices $p_{0}, p_{1}, \ldots, p_{n}$ such that $p_{0}<p_{1}<\ldots<p_{n}$.

The process of defining a flow on $\mathcal{M}(P,<)$ is somewhat convoluted ( $\operatorname{cf}[8, \S 3.2]$ ), but the results of that definition are easily stated. We can define the flow simplex by simplex, in such a way that the flows on two simplices agree on their overlap. We also define $h(t)=\Sigma_{p \in P} n(p) t_{p}$, where $t_{p}$ is the barycentric coordinate of $t$ associated with vertex $p$. We can construct the model $\mathcal{M}(P,<)$ and the flow $\phi: \mathcal{M} \times R \rightarrow R$ such that

- Each simplex is invariant under the flow.

- The vertex set is the rest point set.

- The function $h$ is a Lyapunov function, with $h(p)=n(p)$ for every vertex $p$.

- If $p_{i_{0}}<\ldots<p_{i_{k}}$, then the open simplex $\left(p_{i_{0}} \ldots p_{i_{k}}\right) \subset W^{s}\left(p_{i_{0}}\right) \cap W^{u}\left(p_{i_{k}}\right)$.

An immediate consequence of these properties is: 


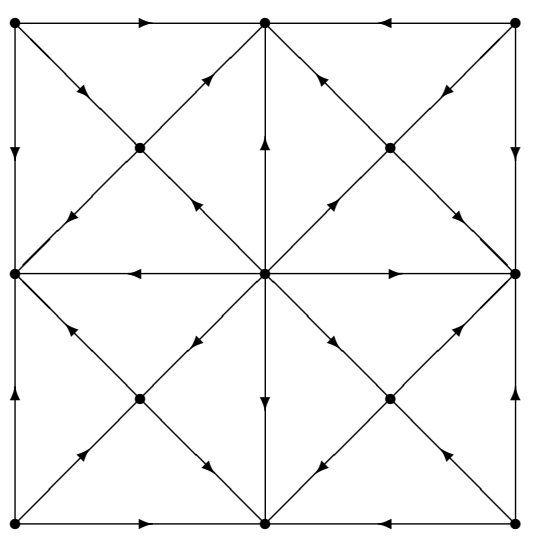

Fig. 1. Flow on the torus

Proposition 3.2. The vertex set $\left\{M_{p}\right\}_{p \in P}$ forms a Morse decomposition for the flow, with flow-defined order $(P,<)$. In particular, each $M_{p}$ is an isolated invariant set.

Not only is the Morse decomposition derived directly from the partial order, but the Conley index of each $M_{p}$ also has natural description in terms of $(P,<)$.

Proposition 3.3. If $\mathcal{M}(P,<)$ is constructed from the partial order of a system satisfying $\mathbf{H 0}-\mathbf{H 3}$, then

- The unstable set $W^{u}\left(M_{p}\right)$ of $M_{p}$ is $M\left(A_{p}^{+}\right)$. This is also the star in $M\left(A_{p}^{+}\right)$of the vertex $p$, and is homeomorphic to the cone on $M\left(A_{p}\right)$.

- $M_{p}$ has the homotopy Conley index of $\Sigma\left(M\left(A_{p}\right)\right)$, the suspension of $M\left(A_{p}\right)$.

- $H_{k}\left(M_{p}\right) \cong \tilde{H}_{k-1}\left(M\left(A_{p}\right)\right)$.

The natural expectation is that $M_{p}$ will have the homology Conley index of $\Sigma^{n(p)}$, which will be the case if and only if $M\left(A_{p}\right)$ is a homology $(n(p)-1)$-sphere. The following example shows that $M\left(A_{p}\right)$ need not be a sphere, nor even a homology sphere. Hence the necessity of $\mathbf{H} \mathbf{4}$ as an explicit hypothesis.

EXAMPLE 3.4. Consider the flow on the 2-torus generated by the following flow on the unit square shown in Figure 1. There are eight Morse sets, each a hyperbolic fixed point, with indices and partial order as illustrated in Figure 2. If $p$ is one of the points of index 2, then $M_{p}$ is the 1-complex shown in Figure 3. This is a wedge of cirles, but not a 1-sphere.

Since we will need to add $\mathbf{H 4}$ as an extra hypothesis, it would be useful if there were a characterization of $\mathbf{H} \mathbf{4}$ in terms of the partially ordered set $(P,<)$. I do not know of such a charaterization, but there are necessary and sufficient conditions for $\mathbf{H} \mathbf{4}$ in terms of $(P,<)$.

Theorem 3. Let $(P,<)$ be a partially ordered set, and $\mathcal{M}(P,<)$ the corresponding complex. 


$$
\begin{aligned}
& n(p)=2 \\
& n(p)=1 \\
& n(p)=0
\end{aligned}
$$

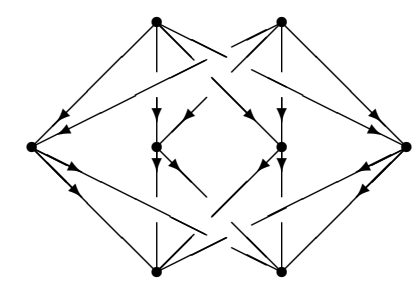

Fig. 2. Partial order of torus flow

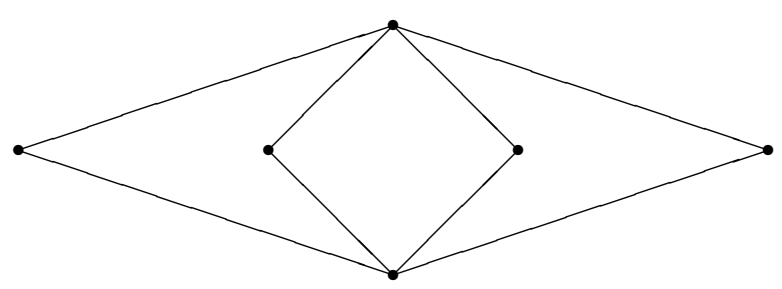

Fig. 3. $M_{p}$ for the repelling points in the torus flow

1. If for every $p$ with $n(p)>0$ there are exactly two $r_{+}, r_{-}<p$ with $n\left(r_{ \pm}\right)=n(p)-1$, then every $M\left(A_{p}\right) \cong S^{n(p)-1}$.

2. If every $M\left(A_{p}\right) \cong S^{n(p)-1}$, then whenever $q<p$ with $n(p)=n(q)+2$, there are exactly two $r_{+}, r_{-}$with $q<r_{+}, r_{-}<p$.

Neither of the converse statements are true.

4. The semi-conjugacy. Having constructed the complex $\mathcal{M}(P,<)$ and its dynamics, we are now ready to define the semi-conjugacy $f: \mathcal{A} \rightarrow \mathcal{M}(P,<)$. There are two basic ingredients to the construction of $f$. First, we choose neighborhoods in $\mathcal{A}$ about the Morse sets $S_{p}$, and define transit time functions $\tau_{p}$ that measure the time an orbit spends in each of these neighborhoods. Next, we construct a Lyapunov function $\Lambda$ on $\mathcal{A}$ that is compatible with these transit time functions. Intuitively, the semi-conjugacy is constructed from these functions in the following steps:

1. An orbit $x \cdot R$ is mapped into the simplex spanned by the points $p \in P$ with $\tau_{P}(x) \neq 0$.

2. Two of the $\tau_{p}$ functions will be infinite; the others will be finite. The finite-valued transit time functions and the Lyapunov function give coordinates that define the image of $x$ in the simplex.

One technicality in this will be reparameterizing the flow on $\mathcal{A}$ to obtain the needed compatibility between the Lyapunov function and the transit time functions. In order for the semi-conjugacy to be well-defined, we require that, if $x, y \in \mathcal{A}$ have $\Lambda(x)=\Lambda(y)$ and $\tau_{p}(x)=\tau_{p}(y)$ for all $p \in P$, then $\Lambda(x \cdot t)=\Lambda(y \cdot t)$ for all $t \in R$. By creating the appropriate subdivisions of $\mathcal{A}$ and adjusting the time each trajectory spends in each subdivision, we can obtain the needed time reparameterization of the flow. 
The use of the transit times in defining $f$ makes it clear that $f^{-1}\left(M_{p}\right)=S_{p}$ and $f^{-1}\left(M_{p}, M_{q}\right)=C\left(S_{p}, S_{q}\right)$. The other features of Theorem 1 follow in a routine fashion. It remains only to show that it is surjective. Since each $M_{p}$ is a point, it is trivial that $f$ maps onto all $M_{p}$. Thus, to show $f$ is surjective, we only need to show that it maps onto each $C\left(M_{p}, M_{q}\right)$. It is at this point that we require the hypothesis $\mathbf{H 4}$. We require this because the "sphericity" provides an algebraic test for surjectivity: if $f: f^{-1}\left(M\left(A_{p}\right)\right) \rightarrow M\left(A_{P}\right)$ is not surjective, then $f_{*}: H_{n(p)-1}\left(f^{-1}\left(M\left(A_{p}\right)\right)\right) \rightarrow H_{n(p)-1}\left(M\left(A_{p}\right)\right)$ is trivial. The proof of Theorem 2 is thus reduced to the algebraic argument that $f_{*}$ is an isomorphism.

5. The hypotheses. I hope that Theorems 1 and 2 will be both useful in their own right, and serve as models for further attempts to realize the Mantra. The applicability of the the theorems depends on the ability to verify the hypotheses, while generalizing the theorem will depend on weakening the hypotheses. I conclude therefore with considerations of these two issues.

5.1. Verifying the hypotheses. To apply this theorem to an attractor $\mathcal{A}$, we must be able to carry out the following computations:

1. Isolate the attractor in $X$.

2. Determine that $\mathcal{A}$ admits a Morse decomposition with index set $P$.

3. Compute the homology Conley index of each of the Morse sets.

4. Compute a connection matrix for the Morse decomposition.

We must further show that the objects identified satisfy the following conditions:

5. The Morse sets must all have the homology Conley index of hyperbolic fixed points.

6. All non-zero entries in the connection matrix must be isomorphisms.

7. If $\Delta_{q p} \neq 0$, then $p$ and $q$ are not adjacent in the flow-defined order.

At this point, the model can be constructed, and the last required condition can be tested:

8. Each $M\left(A_{p}\right)$ must be homeomorphic to $S^{n(p)-1}$.

These eight steps have varying degrees of difficulty associated with them. Assuming the first seven steps have been carried out, the last step is straightforward (with Theorem 3 available to assist). Similarly, if the first three steps have been carried out, verifying (5) and (6) is trivial. Thus, the only steps of any substance are the first four (computing the Conley index information) and the seventh (verifying that the Conley index information has detected all connecting orbits). It is important to note that (7) is fundamentally different than the first four. The first four are purely computational issues, while (7) concerns the ability of those computations to detect the essential dynamical behavior.

The computational issues are considerably easier to deal with, as one of the strengths of the Conley index is its computability. Detecting an attractor and a Morse decomposition, computing the indices of the Morse sets and computing a connection matrix are all well-understood processes. Typically, an attractor is detected by finding a positively invariant neighborhood; a Morse decomposition is detected by a Lyapunov function; homology indices are computed by continuation; and connection matrices are computed by 
the algebraic relations of the attractor-repeller exact sequences. Moreover, the ongoing development of computer-aided Conley index computations [7, 13, 15, 16, 17] promises to make all of these calculations even more tractable, even in cases when the system is only known from experimental data [14].

The real issue, then, is the verification that $S_{p}$ and $S_{q}$ are not adjacent if $\Delta_{q p}=0$. This is emblematic of a much deeper question: does the algebraic information of the Conley index faithfully reflect the dynamical structure of the original system. Clearly, the index information itself cannot answer such a question. Some other form of analysis is required. For these results to be of any practical value, we must be able to carry out that analysis with only partial knowledge of the system. Fortunately, the condition we seek to verify is a negative one: showing that, if $\Delta_{q p}=0$, then $S_{p}$ and $S_{q}$ are not adjacent in the flow-defined order. That is, either there is some $r$ with $q<r<p$, or $p<q$, or $p$ and $q$ are unrelated in the partial order. There are a variety of ways this can be done.

- If $\Delta_{p q} \neq 0$, then $p<q$, so $q \nless p$.

- If there is an explicitly given Lyapunov function $L: \mathcal{A} \rightarrow R$ and $L\left(S_{p}\right)<L\left(S_{q}\right)$, then there can be no connection from $S_{p}$ to $S_{q}$.

- If $n(p)-n(q)>2$ and there are $p_{1}, \ldots, p_{k}$ with $\Delta_{q p_{1}} \Delta_{p_{1} p_{2}} \ldots \Delta_{p_{k} p} \neq 0$, then $q<p_{1}<\ldots<p_{k}<p$, so $p$ and $q$ are not adjacent.

- If all else fails, we must estimate $W^{u}\left(S_{p}\right)$ and $W^{s}\left(S_{q}\right)$, and show that $W^{u}\left(S_{p}\right) \cap$ $W^{s}\left(S_{q}\right)=\emptyset$.

In principle, this is the type of calculation that can be performed numerically, and made rigorous by error estimates. While not an easy matter, such calculations are feasible, particularly if an explicit Lyapunov function is given. The multi-valued map techniques now being developed to carry out the index computations $[7,13,15,16,17]$ may also be used in these calculations.

Once the partial order $(P,<)$ has been identified, the construction of $\mathcal{M}(P,<)$ proceeds in a purely routine fashion. While Theorem 3 does not give a purely graph-theoretic condition for $\mathbf{H 4}$, it does provide tests for $\mathbf{H} 4$ to hold, or to fail. Alternatively, once $\mathcal{M}(P,<)$ is constructed, the verification of $\mathbf{H} \mathbf{4}$ from $\mathcal{M}(P,<)$ is straightforward.

5.2. Necessity of the hypotheses. The conditions are not strictly necessary, in the sense that there are examples in which some or all of hypotheses $\mathbf{H 0}-\mathbf{H} \mathbf{4}$ are not satisfied, but the conclusions of theorems 1 and 2 hold. However, there are also examples that make it clear that some hypotheses of this type are required. In this section, we examine some of these examples and counter-examples. Of course, without hypothesis $\mathbf{H 1}$, the construction is not even defined, so we limit our concern to the other four hypotheses.

First, the invariant set need not be an attractor. Take any compact manifold $N$ with a Morse function. The critical points form a Morse decomposition which satisfies H0-H2. If we limit our attention to a manifold and Morse function that satisfy $\mathbf{H 3}$ and $\mathbf{H 4}$, then there is a semi-conjugacy from $N$ to a model system $\mathcal{M}(P,<)$. Now, embed $N$ as $N \times\{0\}$ in $N \times \mathbb{R}^{k}$, and take a product flow such that $\{0\}$ is repelling in $\mathbb{R}^{k}$. Clearly, $N$ is no longer an attractor in $N \times \mathbb{R}^{k}$, yet the semi-conjugacy still exists. Of course, it no longer 
produces an isomorphism on the Conley indices. If we retain the requirement that the Conley indices are isomorphic, then $\mathcal{A}$ must be an attractor in the ambient space $X$, since $\mathcal{M}(P,<)$ is certainly an attractor in itself.

The hypothesis $\mathbf{H 2}$ is very strong, and there is certainly no reason to expect it to be a necessary condition for the construction of a model and a semi-conjugacy. Indeed, the original paper [9] constructed a model for a system with Morse sets that have the Conley index of a hyperbolic periodic orbit. While that example shows that it is not necessary to assume that Morse sets have the homology Conley index of hyperbolic fixed points, it also suggests why it is natural to make such an assumption.

If $S_{p}$ has a more complicated homology index, we must decide between (at least) two alternatives. On the one hand, we can employ the construction of $\mathcal{M}(P,<)$ used here, which collapses each $S_{p}$ to a point. On the other hand, we may seek to use the homology index to "guess" the appropriate model $M_{p}$ for $S_{p}$, then build the total model $\mathcal{M}$ by collating these model Morse sets. That was the strategy employed in [9]. There we hypothesized that whenever $S_{p}$ had the homology Conley index of a hyperbolic periodic orbit, it had a return map defined on a neighborhood. With this information, it was natural to take a single periodic orbit as the model $M_{p}$.

Obstructions to generalizing this approach are:

- How do we recognize from the homology index what the underlying space should be?

- How do we know what flow to put on that space?

- $\Delta_{q p}$ may now be unreliable as a guide to whether or not $S_{p}$ and $S_{q}$ are adjacent.

- $\Delta_{q p}$ is now a matrix, so there are many different ways that it can be non-zero. How do we interpret these dynamically?

- How do we assemble the model Morse sets to form $\mathcal{M}$ ?

- How do we put co-ordinates on $\mathcal{M}$ so that we can construct the semi-conjugacy?

These obstructions are substantial, and it is not clear that there is any general construction that will successfully deal with all of them. Certainly, [9] suggests that there will be at least some cases that are tractable. Assumptions such as *-hyperbolicity [3] may help to expand that collection. However, if the Morse sets are assumed to have the homology Conley index of a hyperbolic periodic orbit, these obstructions (for the most part) vanish. Obviously, $\mathbf{H 2}$ is not enough to eliminate all difficulties, hence the need for $\mathbf{H 3}$ and $\mathbf{H 4}$. We now turn to a consideration of those hypotheses.

As discussed above $\mathbf{H 3}$ contains the crucial assumption that the algebra of the Conley index detects all connections. To see that this assumption need not always be satisfied, consider the attractor-repeller decomposition of the circle shown in Figure 4(a). The Morse sets are hyperbolic fixed points with $n(i)=i$. Since the index of the total invariant set $S$ is the direct sum of the indices of the Morse sets, the connection matrix must be trivial. That is, the two branches separately have connection homomorphisms that are isomorphisms, but they have opposite orientations and so cancel one another. The algebra provides no evidence of any connections between $S_{1}$ and $S_{0}$. Similarly, in a situation in which $W^{u}(R)$ and $W^{s}(A)$ intersect non-transversely, the connection homomorphism can be trivial. 


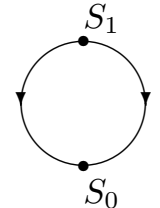

(a)

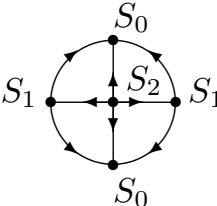

(b)

Fig. 4. Flows on the circle and projective plane

H3 assumes more than just that $\Delta_{q p}$ is non-zero when $S_{p}$ and $S_{q}$ are adjacent. It assumes that $\Delta_{q p}$ is either an isomorphism or is trivial. This need not always occur. Consider the flow on $\mathbb{R} P^{2}$ generated from the flow in Figure 4(b) formed by identifying antipodal points on the boundary. Each of the Morse sets $S_{0}, S_{1}, S_{2}$ is hyperbolic with $n(i)=i$. The connection matrix must compute the homology of $\mathbb{R} P^{2}$ from the chain complex

$$
C_{i} \Delta(P)= \begin{cases}\mathbb{Z} & i=0,1,2 \\ 0 & \text { otherwise }\end{cases}
$$

Clearly, the unique matrix that does this is

$$
\Delta(P)=\left[\begin{array}{lll}
0 & 0 & 0 \\
0 & 0 & 2 \\
0 & 0 & 0
\end{array}\right] .
$$

The first example (i.e. adjacent entries with trivial algebra) appears at this point to be an essential obstruction. If the algebra carrying the dynamical information, there is no reason to expect a model based on the algebra to be meaningful. The second example (i.e. non-trivial entries in $\Delta(P)$ that are not isomorphisms) suggests that a more general construction of the model space may be needed. Suppose we retain H2, and weaken H3 to

H3' There is a unique connection matrix $\Delta(P)$. This matrix has the property that Morse sets $S_{p}$ and $S_{q}$ are adjacent in the flow-defined ordering if and only if the connection matrix entry $\Delta_{q p}$ is non-zero.

If we define $C_{n}=\bigoplus_{p \in P_{n}} C H_{n}\left(S_{p}\right)$ and $\partial_{n}=\Delta\left(P_{n-1}, P_{n}\right): C_{n} \rightarrow C_{n-1}$, then it is natural to interpret the chain complex $\left\{C_{n}, \partial_{n}\right\}$ as the cellular chains of a CW-complex. That is, we might try to construct a CW model instead of a simplicial model for the flow. This is hardly a new idea. After all, Morse theory describes a CW decomposition of a manifold. But, in the Morse theory setting, we start with the assumption of a flow on a manifold. Here, we are starting with an unknown attractor, that looks like a Morse flow on the homology level. Can we, from homological data that emulates that of a Morse flow, construct an actual Morse flow and a semi-conjugacy onto it? This is an open question at present, and will be the subject of future investigations.

Finally, we turn to H4. Example 3.4 shows that $M\left(A_{p}\right)$ need not have the homology of $S^{n(p)-1}$, and so the homology Conley indices of $M_{p}$ and $S_{p}$ need not be isomorphic. 
Some hypothesis of this type is needed. But, could it suffice to assume that $M\left(A_{p}\right)$ is a homology sphere, or a homotopy sphere, to prove that $f$ is surjective? Is the isomorphism of indices required at all for $f$ to be surjective? These are open questions at this point.

\section{References}

[1] M. Carbinatto, J. Kwapisz and K. Mischaikow, Horseshoes and the Conley index spectrum, preprint.

[2] C. Conley, Isolated Invariant Sets and the Morse Index, CBMS Lecture Notes 38 A.M.S. Providence, R.I., 1978.

[3] A. Floer, A refinement of the Conley index and an application to the stability of hyperbolic invariant sets, Erg. Th. \& Dyn. Sys. 7 (1987), 93-103.

[4] J. Folkman, The homology group of a lattice, J. Math. \& Mech. 15 (1966), 631-636.

[5] T. Gedeon, Cyclic feedback systems, to appear in Mem. Amer. Math. Soc.

[6] T. GEDEON and K. Mischaikow, Structure of the global attractor of cyclic feedback systems, J. Dynamics and Diff. Eq. 7 (1995), 141-190.

[7] T. Kaczynski and M. MrozeK, Conley index for discrete multi-valued dynamical systems, Topology Appl. 65 (1995), 83-96.

[8] C. MCCord, Simplicial models for the global dynamics of attractors, preprint.

[9] C. MCCorD and K. Mischaikow, On the global dynamics of attractors for scalar delay equations, Jour. Amer. Math. Soc. 9 (1996), 1095-1133.

[10] K. Mischaikow, Global asymptotic dynamics of gradient-like bistable equations, SIAM J. Math. Anal. 26 (1995), 1199-1224.

[11] K. Mischaikow and Y. Morita, Dynamics on the global attractor of a gradient flow arising from the Ginzburg-Landau equation, Japan J. Indust. Appl. Math. 11 (1994), 185202.

[12] K. Mischaikow and M. MrozeK, Isolating neighborhoods and chaos, Japan J. Indust. Appl. Math. 12 (1995), 205-236.

[13] K. Mischaikow and M. Mrozek, Chaos in the Lorenz equations: a computer-assisted proof, Bull. Amer. Math. Soc. (N.S.) 32 (1995), 66-72.

[14] K. Mischaikow, M. Mrozek, J. Reiss and A. Szymczak, From time series to symbolic dynamics: An algebraic topological approach, preprint.

[15] M. Mrozeк, From the theorem of Ważewski to computer assisted proofs in dynamics, Panoramas of mathematics (Warsaw, 1992/1994), 105-120, Banach Center Publ. 34, Polish Acad. Sci., Warsaw, 1995.

[16] M. MrozeK, Topological invariants, multivalued maps and computer assisted proofs in dynamics, Comput. Math. Appl. 32 (1996), 83-104.

[17] A. SZYMCZAK, A combinatorial procedure for finding isolating neighborhoods and index pairs, Proc. Royal Soc. Edinburgh 127A (1997), 1075-1088. 